\title{
LA REVOLUCIÓN FRANCESA ENTRE LIBERTAD Y TOTALITARISMO
}

\author{
Dr. Gian Battista Bolis Carcassola
}

\section{El concepto "Revolución"}

El uso de "Revolución" como término de significado político es relativamente reciente. Los grandes filósofos griegos, como Platón y Aristóteles, no conocen el significado moderno del tẻrmino. Conciben la transformación de formas de gobierno como fruto de secuencias cíclicas, que implican, esencialmente, cambios en la composición de la clase que gobierna y no alteraciones fundamentales en las esfera social y económica. Igualmente, los grandes historiadores romanos como Tácito y Polibio, dedican su atención sobre todo a los fenómenos de cambio político que nosotros hoy definiríamos "golpes de estado", cambios violentos que conciernen las altas esferas del poder, sin comprometer (en el sentido activo del término) la "masa" y que no producen novedades importantes en las relaciones socioeconómicas.

Igualmente los más importantes pensadores políticos del Renacimiento no conocen la connotación moderna del término "Revolución", y esto no tiene que sorprendernos si pensamos que el más grande entre ellos, Maquiavelo, tiene como fuentes y referencias principales los clásicos latinos, ajenos a los análisis de este tipo de transformaciones políticas'.

En particular hay que recordar que la palabra revolución setá acuñada y usada en el Renacimiento justamente en referencia al lento, regular y cíclico movimiento de los planetas y de las estrellas ${ }^{x}$. Cuando entonces a partir de los siglos XVI y XVII el término Revolución comienza a ser empleado en el campo político, su referencia es la revolución astronómica aplicada a los casos humanos y servirá para indicar la vuelta eterna de algunas formas políticas debidamente inventariadas. Pensadas asi, en referencias al modelo estelar, las revoluciones humanas son vistas como dotadas de caracteres como: la necesidad de volver al

\footnotetext{
'Vease, N. BOBBIO, N. MATTEUCCI, G. PASQUINO Dizionario di Politica - Voz "Revolución", Torino, 1990.

${ }^{2}$ En este campo, tevolución significa movimiento que conduce las cosas a su punto de partida.
} 
estado anterior, la inevitabilidad, la regularidad, el orden, la pasividad de los hombres que observan cambios en los cuales es ausente totalmente la novedad.

En el siglo XVIII la concepción no varia y la revolución es "en primer lugar el retorno de formas ya aparecidas", pero, paralelo a este concepto de revolución se iba difundiendo otro más popular, vulgarizado, del cual dan testimonio los diccionarios de la época: revoluciones como vicisitudes de la existencia humana, caracterizadas por cambios extraordinarios ocurridos por asuntos políticos, reveses de fortuna de las naciones. Se trata ya no del retorno al orden, sino del surgimiento imprevisible y brusco de algo nuevo, donde prima el desorden con respecto al orden antiguo.

Los dos significados; culto y vulgarizado, se encuentran también en libros importantes del siglo XVIII como "Droits et Devoirs du Citoyen" de G.B. Mably de 1750. Ya se habian encontrado ocasionalmente esfuerzos negociadores entre orden y extraordinario, como es el caso de J. Benigne Bossuet en su "Discurse sur l'Histoire Universelle" de 1691.

\section{Su connotación moral}

Así como no se tiene una idea clara del término revolución en el campo político, tampoco es clara su connotación moral. Las revoluciones, para el siglo XVIII, son buenas o malas, llevan el progreso o la regresión; pero una cosa es cierta: por lo general, los hombres las temen, porque producen inestabilidad política y ponen en evidencia la dificultad humana a controlar los acontecimientos. En visperas de la Revolución Francesa no era claro "ni que fuese una revolución en campo sociopolítico, ni se auspiciaba o concebía que los hombres puedan iniciar una revolución" ". Mas, la Revolución Francesa, desde su comienzo, nos sorprende con un carácter espectacular, insólito, que llena a los contemporáneos de estupor (la literatura de la época tiene abundantes testimonios de este asombro) y la primera consecuencia de esto es que nadie se pone el problema de si se trata de una restauración o instauración, mas bien la Revolución Francesa vive de la idea que lejos de reanudar las cadenas del tiempo, constituye un comienzo absoluto, una época nueva, una nueva "edad de oro". En su aurora, como bien ha visto J. Michelet la Revolución Francesa se piensa y se

\footnotetext{
${ }^{3}$ M. OZOUF, Diccionario de la Ravolución Framcesa Madrid, 1989: 692

${ }^{4}$ Nótese a este propósito la concordancia con la Revolución Rusa asi como ha sido ilustrada por autores como M. HELLER y V. STRADA, "Francia e Rivoluzione Russa. Analogia Revolucionaria" L'eredita dello Rivaluzione Francese e Russa edicada pot F. FURET, Bari 1989: 221-246.

"t"Desde sus primeros pasos la revolución se piensa como la liberación de rodos los modelos históricos" M. OZOUF, Dicionario de la Revolución Francesa: 694.
} 
vive como salida de la historia; para la revolución el tiempo no existía, había perecido. Existen historiadores que se ponen frente a la Revolución Francesa con espíritu crítico, tratando de examinarla con los documentos y los hechos reales, pero existen todavía muchos, y a veces son los "grandes" que no aceptan esto, ni lo desean. Es esta última, por ejemplo, la postura de Jacques Le Goff, el conocido estudioso francés de la Edad Media, que ve con dolor el nacimiento de una corriente historiográfica "contrarevolucionaria" que define "de pseudohistoriadores", diletantes e incompetentes, sin rigor científico. Hasta aquí uno podía seguirlo, porque el mundo está lleno de trabajos pseudo-científicos; pero si profundizamos y estamos atentos cuando comienza a hablar de los contenidos de estas investigaciones pseudo-científicas, nos damos cuenta que su invectiva acaba para incluir aquellos historiadores que han tenido el valor de indagar, por ejemplo, sobre el genocidio o "pueblicidio" de la Vendée. Además podemos fácilmente detectar su parcialidad cuando nos da una definición de la Revolución Francesa como "símbolo auténtico, nacional, europeo, universal que es aún hoy de esperanza", o cuando acaba por decir: "tengo ganas de gritar: no toquéis mi revolución".

Desmitificar la Revolución Francesa no es fácil, porque este mito tiene raíces muy profundas y se puede decir que ha comenzado con la misma revolución. También antes de la Revolución Francesa había formas de organización de la opinión pública, por ejemplo, todo el aspecto espectacular de las ceremonias de ingreso de los reyes en las ciudades, los autos de fe, etc... pero ahora las cosa son diferentes, "científicas": la revolución celebra a sí misma, promueve fiestas, no teme en alterar la verdad acentuando unos aspectos y eliminando otros. Se sirve en manera masiva de la difusión de folletos, litografias, afiches'.

En segundo lugar, el "mito" de la Revolución Francesa se ha fortalecido en el siglo XIX por el hecho de que muchos historiadores (franceses sobre todo) lo han tratado como punto de referencia de los acontecimientos sucesivos, como paradigma de la historia contemporánea. Así, se han identificado las nuevas etapas históricas como el repetirse más lento de las diferentes fases de la Revolución: el 1830 se ha asimilado al 1789 , el 1848 al 1792-94, la elección de Bonaparte a la Reacción Termidoriana, el segundo imperio de Napoleón III al primero, hasta

\footnotetext{
1). MICHELET, Histoine de lo Renolwtion Frantaise Paris, Gallimard, Bibl. de la Pléiade, 1939, t. 1, p. 12.

'Este papel jugado por la imprenta en la Revolución Francesa no sólo es notabie en relación a su autocelebración frenté a la historia, sino en las disputas y batallas por el poder "dentro de la misma revolución"; Robespietre, por ejemplo no se dirige en la Asamblea Constituyente a sus colegas sino de las tribunas al "pueblo".. Sus discursos son conocidos de antemano en todas las tabernas de la capital.
} 
transformarse (la revolución francesa) en "emblema" de la Francia, en algo sagrado $^{8}$.

Mas, si para algunos la Revolución Francesa es algo que no se "puede tocar", para otros comienza a ser una herencia fastidiosa, vergonzante. Los partidos comunistas europeos comenzaron a distinguir entre unos momentos y otros de revolución?. Interesante es también la postura de otros intelectuales de área socialista ${ }^{10}$ tuvo ocasión de escribir en 1989 que no había que festejar la Revolución Francesa porque ha sido un monstruoso error de base que no ha sido detectado a tiempo y así ha fomentado un pensamiento político nefasto, al cual se deben los errores de la Revolución Soviética y de todo revolución, hasta Khomeini. El artículo concluye, después de haber dicho que con la revalución los franceses han dado un mal ejemplo a toda la humanidad, diciendo "nuestros padres son Locke, Montesquieu, los ilustrados y no los revolucionarios franceses". Se trata aquí de otro tipo de operación cultural bien conocida. Separar la Revolución Francesa de la Ilustración echando las culpas de los "desastres" de la revolución a los mismos actores inmediatos y "salvando" la ideología iluminista.

Es la misma postura de otros intelectuales socialistas ${ }^{11}$ al afirmar que las bases de la Izquierda Unida Europea se puedan encontrar sólo en un retorno a la Ilustración. Asistimos así a una segunda perspectiva: los nuevos iluministas que tratan de alejarse de la Revolución Francesa vista como una herencia pesada. Esto permite que el mito entre en crisis y comience a salir a flote la verdad histórica. Pero es necesario precisar dos puntos fundamentales:

a. El mito ha comenzado a entrar en crisis y está efectivamente en crisis en el campo de los especialistas; pero todavía muy poco de esto se advierte en los libros escolares que conservan el esquema clásico de la Revolución como aurora de la humanidad.

b. De todas formas no se puede olvidar que la Revolución Francesa ha sido un elemento decisivo en la historia occidental produciendo una ruptura total con el pasado y el comienzo de una nueva época. A

\footnotetext{
"En la Sorbona de París existe (y tiene más de cien años), una cảtedra de "Historia de la Revolución Francesa" y, en esto, es fácil nuevamente observar las similitudes con la Revolución Rusa.

'Es el caso del Ex-Partido Comunista Italiano que por boca de su secretario en 1989 ha afirmado que ellos no quieren referirse al 1792 sino sólo al 1789 .

${ }^{10}$ Fue el caso del sociólogo y politólogo italiano muy conocido en los años ' 80 Fíancesco Alberoni, en la columna del diario itsaliano más leído, el Corriene delle Sera.

"Como Luciano Pellicani, director de la revista socialista Mondo Opentrio, que tuvo un cierto peso en el socialismo europeo de los años ' 80 .
} 
partir de la noche del 4 de agosto (fecha mucho más significativa que el 14 de julio), inicia un proceso revolucionario que en cierto sentido no ha terminado, porque teniendo como objetivo la realización de una utopia, no se ha realizado todavía y no se realizará. Este carácter de novedad ha sido bien evidenciado en 1790 por E. Burke en su obra "Revolution" en la cual define la revolución como un hecho inaudito y asombroso en cuanto subvierte el orden del mundo, que, de lo contrario, la revolución inglesa trataba de reflejar. Burke es particularmente icástico cuanto afirma que la Revolución Francesa ha terminado la época de la caballería en Europa y ha comenzado en la época de los contables, intuyendo ya desde el comienzo (1790) la desembocadura jacobina, terrorista y totalitaria del 1792. En realidad los que contraponen 1789 y 1792 lo hacen muchas veces con una falta de respeto por la sucesión cronológica de los hechos.

\section{Una guerra religiosa}

Desde este punto de vista es emblemática la interpretación de las relaciones Iglesia Católica - Revolución Francesa. Se ha difundido la idea que si bien los revolucionarios han sido a veces exagerados ${ }^{12}$, pero estarian justificados porque habrian golpeado la iglesia en cuanto ligada a la Monarquia y a la Aristocracia y porque su batalla se dirigía contra los "signos exteriores del cristianismo". La afirmación merecería la sontisa que provocan las cosa ridículas si la realidad histórica no fuera tan trágica. Las decenas de millares de sacerdotes, religiosos y religiosas asesinados ¿'son "signos exteriores"? Las miles de iglesias, monasterios y conventos saqueados, cuando no destruidos ¿cómo los debemos considerar?

Pero no es sólo eso, hay un argumento histórica muy ponderado contra esa justificación: La Revolución Francesa ha sido antimonárquica sólo en una segunda fase. Se piensa que todavía en 1791 uno podía ser expulsado del club de los "jacobinos" si se declaraba en contra de la monarquía; mientras que desde el comienzo han sido publicados un sinnúmero de libros, periódicos, panfletos, afiches contra la iglesia, los mismos que exaltaban al Rey y aceptaban la aristocracia. Hacia julio de 1791 , después del intento de huida del Rey, la revolución no es antimonárquica, mientras es verdadera la relación opuesta: es el Rey que será combatido con mayor vehemencia cuando a un cierto punto tratará

\footnotetext{
"Es interesante sobre este punco buscar la voz "vandalismo" en el Dixcionario de la Revolución Francese de FURET-OZOUF
} 
de oponerse a la persecución de la iglesia. Interesante desde este punto de vista, y poco conocido en ambientes no especializados, es el testamento del rey, en el cual afirma que su "único verdadero remordimiento es haber dado su consentimiento a la Constitución Civil del Clero", asi como de relieve es la muerte del rey, que hace decir a autores de área socialista como Furet y Ozouf que "si bien su educación cristiana y real no le habia proporcionado los medios para una defensa, pero le habia enseñado a morir, lo cual hizo como un rey muy cristiano, transformando asî el reicidio en deicidió" ${ }^{\prime 13}$. No se puede entonces, opinar que la iglesia fue perseguida porque se puso al lado de los aristócratas; entre las víctimas de la revolución el porcentaje de los aristócratas no es superior al porcentaje de los mismos de la sociedad. Es verdad, la revolución se dice a cada instante antiaristócratas, pero de una manera especial: al enemigo se le aplica un nombre infamante como en otras épocas: "enemigos del pueblo", "fascista", etc.- y con esto se ha encontrado la razón para eliminarlo. Por eso es posible encontrar con frecuencia que durante la revolución se definan con el término de "aristócrata"los que antes eran "revolucionarios" y ahora han caído en desgracia.

En realidad, el proceso de descristianización comienza desde 1789, mucho antes de la condena de Pío VI, pronunciada el 10 de octubre de 1791, después de la aprobación por parte de la revolución de la Constitución Civil del Clero. Ha comenzado con la supresión del diezmo (el 4 de agosto de 1789) y se encarnizó el 2 de noviembre expropiando todos los bienes de la iglesia, hecho que constituyó, a juicio de muchos historiadores, un golpe mortal en el sistema educativo, asistencial y sanitario de Francia, empobreciendo el pais sin solucionar el problema del déficit del Estado ${ }^{14}$. Es importante escuchar lo que dice Pierre Chaunu a este propósito, sobre todo si se tiene en cuenta que se trata de un historiador calvinista no ciertamente favorable a la Iglesia Católica. Pierre Chaunu, con su estilo fuertemente polémico, define a los revolucionarios como "irresponsables, desenfrenados", gente que "no entendia nada de economía" y que "sólo tenían claro que los que tenían que pagar eran los otros". Su análisis continúa diciendo: "Así empezaron a buscar lo que podian confiscat: en primer lugar suprimen el diezmo de iglesia, que nadie del pueblo pedía suprimir porque significaba quitar las subvenciones a las escuelas y a los hospitales. Luego se confiscan los bienes de las abadías" ${ }^{\text {"15 }}$. Después viene la supresión de las órdenes religiosas y en fin la Constitución Civil del Clero, la cual así habla el mismo Pierre Chaunu: "Se impone la Constitución Civil del clero, pues sin modificar y emancipar la estructura de la iglesia no habrían podido robar los bienes de la iglesia, que desde siglos mantenía escuelas y hospitales, son acaparados por una

\footnotetext{
"FURET-OZOUF Discionario de la Revolusión Francesa: 121.

"F, FURET, D, RICHET, La Rivoluzione Francese Bari, 1987:151-157.

P. CHAUNU, Entrevista al semanario I/ Subato Roma 29/04/1989.
} 
banda de ochenta mil familias de ladrones, nobles, burgueses, de derecha y de izquierda. ¡Es por esto que todavía hoy en Francia la Revolución es intocable! Porque fue un gran robo con ventaja para la clase dirigente. EI robo necesita de la mentira y de la persecución porque no es fácil imponer el abuso a los curas y al pueblo. Por esto quiso imponer el juramento de los curas y quien no juró fue asesinado. La revolución ha sido una guerra de religión".

No se trató entonces de una resistencia de la iglesia a renunciar de sus privilegios, mas bien del intento por parte de los revolucionarios de moldear una iglesia a su imagen y semejanza, hecho que no se dio.

\section{Libertad, Igualdad y Fraternidad}

Si es verdadero lo que acabamos de decir, ¿Cómo entender los slogans de la Revolución Francesa que han llenado los oídos de Europa y del mundo con un grito de novedad y liberación? - ¿Cómo conciliat su actitud tan violenta con estas famosas palabras?

Además muchos historiadores, hombres de cultura y hasta el mismo Juan Pablo $\mathrm{II}^{16}$ han puesto el acento sobre las raíces cristianas de las tres famosas palabras: "Liberté, Egalité, Fraternité". Esto es verdadero y entonces, aqui se impone un análisis crítico que nos ayude a comprender lo que ha sucedido o, como dice Chesterton porque estas "verdades se han enloquecido".

En primer lugar la palabra libertad. Probablemente el punto más fácil de explicar y el más estudiado. Ya Marx y Engels en el "Manifiesto del Partido Comunista" hacen una aguda crítica a la burguesía, diciendo que en su llegada al poder ha disuelto la Civilización Feudal con sus privilegios pero también con sus relaciones, aislando la persona, volviéndola individuo solo frente al estado, quitándole el aspecto sagrado del trabajo y de la profesión. Para Marx la Revolución Burguesa ha provocado la concesión Formal a todos los hombres de las libertades políticas, sin saber sustanciar estas libertades en un contexto social capaz de garantizar el efectivo ejercicio.

Estas afirmaciones contienen un núcleo de verdad que vale la pena desarrollat.

\footnotetext{
th Homilia del $1^{\circ}$ de junio de 1989 en Bourger Francia.
} 
El Antiguo Régimen, más que la "Libertad" conocía "las libertades"; las libertades francesas habian sido ligadas a una historia de siglos, fundadas en autonomias, privilegios, hasta usurpaciones, pero cosas reales, eran derechos de los cuales los Municipios, las Órdenes, las Universidades, la que hoy llamariamos "cuerpos intermedios", "grupos de opinión" o con un término más comprensivo "Ia sociedad civil" gozaban parecidas libertades estaban ancladas a la complejidad de la sociedad; de lo contrario la libertad proclamada por la Revolución Francesa, es abstracta y puede desarrollarse sólo en una unidad y homogeneidad total. Es una "idea pura", pura abstracción con la cual se puede entrar en contacto a través de palabras y no en la realidad. Íntimamente ligados a esta concepción abstracta de libertad están los conceptos de "unanimidad" y "voluntad general", ya presentes en la obra de los constituyentes. No es necesario llegar a Robespierre; el terror esta íntimamente ligado a está concepción abstracta de libertad que, teniendo un carácter utópico -y por eso irrealizable- para seguir prosperando tiene que justificar sus fracasos echando a otros la culpa; por eso continuamente inventa culpables, enemigos. Rousseau habia difundido la idea que "el hombre nace bueno y la sociedad lo corrompe" y Voltaire había acusado del mal presente a los "siglos pasados de superstición e ignorancia". Los hombres de la Revolución, también ellos están convencidos que una vez quitadas las trabas del pasado será posible consolidar una sociedad perfecta.

En este sentido son importantes las actitudes de los revolucionarios con respecto a la educación. Una ley del año II sobre la reforma de la instrucción, por ejemplo, prevé la abolición de la enseñanza de la historias en las escuelas primarias, con la motivación que la historia no tiene nada que enseñar, de lo contrario representa algo de lo que hay que liberarse. Otro episodio paradigmático es lo del día del asesinato de Marat (13 de julio de 1793) Robespierre interviene y presiona a la convención - aprovechando del clima de particular tensión dramática- porque adopte el proyecto de ley de la Reforma de la Educación elaborado por Lepeletier de Saint Fatageau, que prevé la "regeneración" de los jóvenes a través de un "molde republicano" consistente en quitar a los niños a sus familias y encerrarlos en internados llamados "casas de igualdad". Hay que decir que, por suerte, la curación no durará más de unos meses... Pero ¿qué pasa, si a pesar de todos los esfuerzos la sociedad perfecta tarda en nacer y en su lugar comienzan a brotar dificultades, escasez, hambre? He aquí el refrán de los "enemigos de la revolución" y "traidores".

En este momento la libertad teóricamente proclamada para todos es puesta en discusión. La libertad es para "todos los buenos revolucionarios", no hay libertad para los "enemigos de la revolución". Chabot repetia que opuesto a

"1. BACHELER, En A. COCHIN, Lo Spirito del Giacobiniamo, Milano 1989:22-23. 
un pueblo unido no hay más que un solo partido: el de los "conspiradores", y es un conspirador quien se sale de un marco trazado. ¿Qué sucede si un pueblo se resiste, si no responde? Otro revolucionario, Billaud Varenne, responde: "hay que restituir el pueblo a la libertad". Se puede observar aquí una tendencia típica de la revolución; hacer de los hombres lo que el legislador (el poder) quiere que sean. La revolución pretende determinar las vidas de las personas en todos los aspectos; se dan leyes relativas a los más diferentes aspectos y campos de la vida, constituyendo así una especie de "pedagogía diaria" y ese fenómeno no se limita a la época del Terror de lo contrario aumenta a partir de Termidoro.

El principio de la libertad se relaciona a aquel de la igualdad. Hay obligación de hacer en modo que todos los hombres sean absolutamente iguales. Condorcet, sostiene que la igualdad es el fin último del "arte social". Se trata de una lucha contra "todo tipo de inigualdad", por esto hay que borrar todos los recuerdos del pasado.

Emblemático es el caso del calendario, donde el deseo de igualdad empuja el Romme a proponer dividir el año en meses con el mismo número de días, a prever años siempre iguales (sin bisiesto) ${ }^{19}$. Al final en el Calendario Revolucionario desaparecen los Domingos y los Santos, los nombres de los meses y serán sustituidos con nombres de la naturaleza de las operaciones agrícolas de los "bienhechores de la humanidad" Tanta "innovación" duró muy poco, ya que en 1806 se revocan estas medidas. Muy diferentes es el destino de los Departamentos, creados al comienzo del proceso revolucionario y otro ejemplo emblemático del concepto de igualdad. Todas las regiones históricas de Francia fueron borradas junto a sus diferencias jurídicas e institucionales y en su lugar se crearon los departamentos.

Burke es el primero en comentar que "fue la primera vez que en la historia se veían a unos hombres, despedazar su Patria de forma tan bárbara".

Los departamentos tenían que ser iguales y hubo discusiones por parte de Mitaneau y los fisiócratas acerca de si tenían que ser iguales por tamaño o por población. Al final prevaleció el criterio de la superficie, adoptando una

\footnotetext{
"Se dan leyes sobre el comportamiento en público, sobre "como" hay que llevar las insignias, el ripo de vestimentas, como "tutearse",

${ }^{19}$ El Romme llega a proponer días de 10 horas y se produjeron por un cierto tiempo relojes diézmales que ahora constiruyen una curiosidad para los coleccionistas.

${ }^{3}$ En cl caso de los meses la propuesta de los hombres naturales prevaloció sobre la propuesta del Romme de nombrar los meses con eventos propios de la Revolución, como: mes de la Bastilla, del Pueblo, etc.
} 
corrección para la proporción de los representantes que tendrá en cuenta el número de habitantes.

En fin, la palabra fraternidad es la hermana menor y la menos importante de las palabras revolucionarias, no se encuentra en los "Cahiers des Doleances" de los primeros tiempos revolucionarios, mas bien hace su aparición en las Fiestas Revolucionarias que sustituyen los cultos religiosos y en los "clubes" donde los afiliados se saludan en nombre de la Fraternidad. Es importante recordar que el sentido de la palabra es muy distinto al sentido cristiano, mas bien guarda cierta similitud con la hermandad masónica. En el fondo se trata de una fraternidad que excluye a algunos, lo que importa a los revolucionarios es desenmascarar a los "falsos hermanos".

Dr. Gian Battista Bolis Carcassola Unîversidad Católica Sedes Sapientiac 